\title{
Tisagenlecleucel in Non-Hodgkin Lymphoma: The Restricted Mean Survival Time as a Tool for Estimating Progression-Free Life Expectancy Better than the Median
}

\author{
Marco Chiumente $^{a} \quad$ Daniele Mengato $^{b} \quad$ Andrea Messori $^{c}$

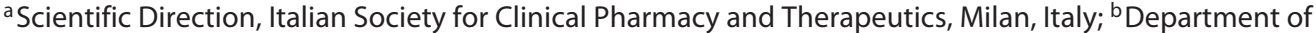 \\ Hospital Pharmacy, Bolzano Central Hospital, Bolzano, Italy; ${ }^{C}$ HTA Unit, Regional Health Service, Florence, Italy
}

\section{Dear Editor,}

Although the restricted mean survival time (RMST) is considered a new methodological tool for interpreting survival curves, its development dates back to more than 15 years ago. In comparison with the median, the RMST has a specific advantage because it examines the entire survival curve (as the hazard ratio) and expresses the survival outcomes using time as a unit of measurement (as medians). More importantly, the RMST captures the presence of a long-term survival plateau, which of course reflects a better prognosis. Most previous experience in the application of RMST is focused upon oncology [1], but other fields are being investigated as well [2]. An original method of calculation, drawn from the field of pharmacokinetics, has markedly simplified the otherwise complex estimation of RMST [3, 4].

In the present analysis, we assessed the RMST from the progression-free survival curve of the patients enrolled in the phase- 2 one-arm JULIET trial [5]. Our objective was simply to compare the RMST with the median reported by the JULIET investiga-

The authors contributed equally to the study. tors and to determine the ratio between these two parameters

The patient group treated with tisagenlecleucel consisted of 48 patients. Their inclusion criteria were diffuse large B-cell lymphoma, at least two previous lines of treatment, including rituximab and an an-

thracycline. Autologous stem cell transplantation had been performed in $49 \%$ of these patients.

According to our analysis, the value of RMST in the progression-free survival curve (Fig. 1) was 7.74 months ("milestone" set at 18 months of follow-up). The

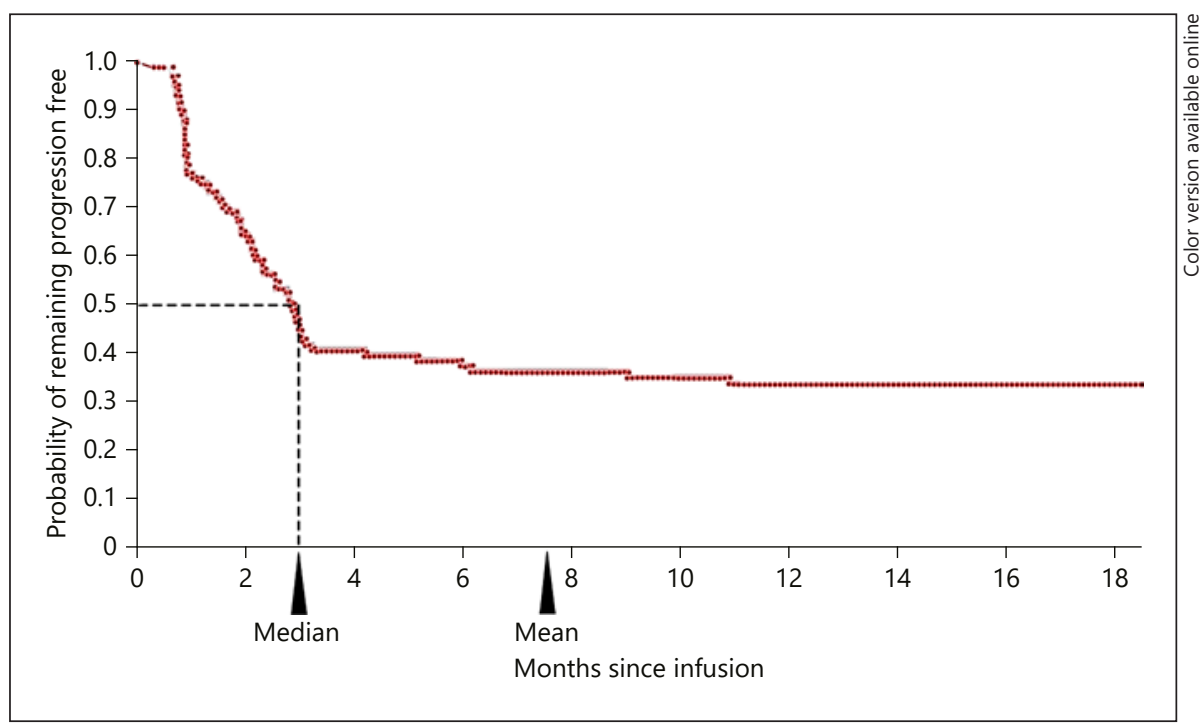

Fig. 1. Progression-free survival curve in the JULIET trial. Value of RMST estimated by model-independent methods and the difference between the median and mean. karger@karger.com

(C) 2020 S. Karger AG, Basel

www.karger.com/aha

Karger
Marco Chiumente, PharmD, Scientific Direction

Italian Society for Clinical Pharmacy and Therapeutics

Via Carlo Farini 81

IT-20159 Milan (Italy)

marcochiume@yahoo.it 
corresponding median published in the JULIET trial was 3.0 months. Hence, the $\mathrm{RMST} /$ median ratio was 2.58 . The milestone is the point in time in the follow-up at which the area under the survival curve is truncated.

The further follow-up in the JULIET trial, which is eagerly awaited, will allow to set a milestone at more than 18 months and to re-determine the RMST. The obvious

\section{References}

1 Trinquart L, Jacot J, Conner SC, Porcher R. Comparison of Treatment Effects Measured by the Hazard Ratio and by the Ratio of Restricted Mean Survival Times in Oncology Randomized Controlled Trials. J Clin Oncol. 2016 May;34(15):1813-9.

2 McCaw ZR, Yin G, Wei LJ. Using the Restricted Mean Survival Time Difference as an Alternative to the Hazard Ratio for Analyzing hypothesis is that, with a longer follow-up, the rate of RMST/median could be increased to a greater degree.

In conclusion, the present analysis confirms the usefulness of the RMST in handling survival curves, particularly when the final portion of the curve shows a plateau. The main advantage consists in providing a numerical estimate of survival that captures the presence of the plateau.

Clinical Cardiovascular Studies. Circulation. 2019 Oct;140(17):1366-8.

3 Damuzzo V, Agnoletto L, Leonardi L, Chiumente M, Mengato D, Messori A. Analysis of Survival Curves: Statistical Methods Accounting for the Presence of Long-Term Survivors. Front Oncol. 2019 Jun;9:453.

4 Messori A, Damuzzo V, Agnoletto L, Leonardi L, Chiumente M, Mengato D. A model-

\section{Disclosure Statement}

The authors have no conflicts of interest to declare.

\section{Funding Sources}

The authors did not receive any funding.

independent method to determine restricted mean survival time in the analysis of survival curves. SN Compr Clinl Med. DOI: 10.1007/ s42399-019-00199-7.

5 Schuster SJ, Bishop MR, Tam CS, Waller EK, Borchmann P, McGuirk JP, et al.; JULIET Investigators. Tisagenlecleucel in Adult Relapsed or Refractory Diffuse Large B-Cell Lymphoma. N Engl J Med. 2019 Jan;380(1):45-56. 